\title{
Meso Scale Discovery and Luminex Comparative Analysis of Calbindin D28K
}

\author{
Samer Sourial, Maritha Marcusson-Ståhl, and Karin Cederbrant \\ Molecular Toxicology, Safety Assessment, AstraZeneca R\&D Södertälje, 15185 Södertälje, Sweden \\ Correspondence should be addressed to Samer Sourial, s.sourial@student.liverpool.ac.uk
}

Received 6 May 2009; Accepted 23 July 2009

Recommended by Colin Cooper

\begin{abstract}
The sensitivity of different renal regions to xenobiotics requires the development of a multiplex immunoassay for the simultaneous analysis of kidney biomarkers. Calbindin D28K is a distal tubule-specific protein that can be detected in urine under pathological conditions. In this study, a pair of anti-calbindin D28K antibodies was used in an immunoassay for the detection of calbindin D28K expression in rat and human kidney and urine. Comparative analysis of the immunoassay was performed on the Meso Scale Development (MSD) and Luminex platforms. Analysis on both platforms detected calbindin D28K concentrations between $100 \mathrm{ng} / \mathrm{mL}$ and $100 \mathrm{pg} / \mathrm{mL}$. Luminex detected 10-fold the amount of calbindin D28K in samples analyzed as compared to MSD, whereas calbindin D28K level in rat and human urine was below detection limit in both platforms. The application of the immunoassays described herein may be useful in toxicological and pathological studies of distal tubular damage in rats and human.
\end{abstract}

Copyright (๑) 2009 Samer Sourial et al. This is an open access article distributed under the Creative Commons Attribution License, which permits unrestricted use, distribution, and reproduction in any medium, provided the original work is properly cited.

\section{Introduction}

The high renal blood flow, renal biotransformation of chemicals to reactive metabolites, and the nephrons ability to concentrate tubular fluid render the kidney sensitive to xenobiotics. Due to the kidneys regional sensitivity to xenobiotics, it is important to colocalize sites of biomarkers release with pathological lesions [1].

Calbindin D28K is an intracellular, vitamin D-dependent, calcium-binding protein that is expressed in the epithelial cells of renal distal tubules [2, 3]. Previous studies have demonstrated the inhibitory effect of nephro-toxicants such as Cyclosporin A on the expression of calbindin D28K in distal tubules [4]. Furthermore, urinary analysis of patients treated with Cisplatin as well as patients treated by extracorporeal shock wave lithotripsy has indicated that the expression of calbindin $\mathrm{D} 28 \mathrm{~K}$ can be increased under pathological conditions $[5,6]$. The specific localization of calbindin $\mathrm{D} 28 \mathrm{~K}$ in distal tubules along with its pathophysiological release in urine makes this protein a potential biomarker for distal tubule damage.

There is an emerging trend in pharmaceutical industry to evaluate biomarkers to determine the safety of drugs early in clinical development. These biomarkers should predict specific damage before functional loss. Limited sample volume and excess costs have led to the development of multiplex immunoassays for the simultaneous analysis of multiple biomarkers.

The aim of this study was to compare an immunoassay for calbindin D28K analysis on two multiplex platforms. The same antibody pair was used for the single-plex analysis of calbindin D28K in Luminex as compared to Meso Scale Development (MSD). Analyses were performed using rat recombinant calbindin D28K protein as a standard. Kidney homogenates were used for validating the immunoassay on both platforms. Results from this work may provide clues to the further development of a multiplex immunoassay for the simultaneous analysis of multiple kidney biomarkers.

\section{Materials and Methods}

2.1. Sample Preparation. Rat and human homogenates were kindly provided by AstraZeneca (UK) and Cardiff university (UK), respectively. Briefly, frozen tissue samples were chopped into small cubes (approximately $1 \times 1 \times 1 \mathrm{~mm}$ ) and homogenized in RIPA buffer (New England Biolabs, Hitchin, UK) containing Halt protease inhibitors (Pierce, Rockford, 
ILL, USA) using a Polytron (Kinematica, Bohemia, NY, USA) homogenizer. Samples were homogenized for two bursts of 30 seconds and placed on ice for one minute in between bursts. Samples were then centrifuged at $10000 \times \mathrm{g}$ for 5 minutes to remove insoluble debris. Frozen urine samples from healthty rats and human individuals were used in this study.

2.2. MSD Immunoassay. Anti-mouse immunoglobulin plates (MSD, Gaithersburg, Md, USA) were used to immobilize anti-calbindin D28K monoclonal antibody from murine ascetic fluid (Sigma, Saint Louis, Mo, USA). The monoclonal antibody was diluted in Tris-Base Saline (TBS) buffer to $5 \mu \mathrm{g} / \mathrm{mL}$ and $25 \mu \mathrm{L}$ was added per well. The plate was incubated overnight at $4^{\circ} \mathrm{C}$ and each well was blocked with $25 \mu \mathrm{L}$ of $5 \%$ Blocker-A (MSD) in TBS. After one hour of incubation in blocking buffer, the plate was washed three times with $0.1 \%$ Tween-containing TBS (wash-buffer). All incubations in the MSD immunoassay were done on an orbital shaker at $650 \mathrm{rpm}$. Rat recombinant calbindin D28K (Swant, Bellinzona, Switzerland) was used as a standard and a dilution series was prepared in dilution buffer, starting at $500 \mathrm{ng} / \mathrm{mL}$. Throughout the MSD immunoassay a buffer containing 2.5\% Blocker-A dissolved in TBS was used as a dilution buffer. Duplicates of both standard and samples were analyzed and $25 \mu \mathrm{L}$ were pipetted per well. After one hour of incubation, the plate was washed three times with wash-buffer and $25 \mu \mathrm{L}$ of anti-calbindin D28K polyclonal rabbit antibody (Sigma, Saint Louis, Mo, USA) was added per well. The polyclonal antibody was diluted to $2 \mu \mathrm{g} / \mathrm{mL}$ in dilution buffer and $25 \mu \mathrm{L}$ was added per well. After one hour of incubation, plate was washed three times with wash-buffer and $25 \mu \mathrm{L}$ of sulfo-tagged goat anti-rabbit antibody (MSD), $2 \mu \mathrm{g} / \mathrm{mL}$, was added per well. The plate was then covered from light while incubating for 30 minutes and then washed three times with wash-buffer. Finally, $150 \mu \mathrm{L}$ of $2 \mathrm{x}$ MSD read buffer $\mathrm{T}$ was added per well and the plate was measured on the MSD Sector Imager 6000. The raw data was analyzed using the default setting of the Discovery Workbench 3.0 software (MSD).

2.3. Luminex Immunoassay. Anti-mouse IgG1 beads (Upstate, Temecula, Calif, USA) were used to capture anticalbindin D28K murine antibody. The beads were vortexed vigorously for one minute and diluted 1:40 in TBS. The plate was pre-wet with $150 \mu \mathrm{L}$ TBS for 10 minutes and then aspirated using a vacuum manifold with the bottom of the plate blotted on paper towel to remove excess fluid. Throughout the assay the beads were kept protected from light and the incubations were performed on an orbital shaker at $650 \mathrm{rpm}$. The diluted beads were vortexed for one minute and $25 \mu \mathrm{L}$ was added per well followed by $25 \mu \mathrm{L}$ of anti-calbindin D28K murine antibody diluted in TBS to a concentration of $5 \mu \mathrm{g} / \mathrm{mL}$. After incubating for one hour, the fluid was removed using a vacuum manifold and the wells were washed twice with $0.1 \%$ Tween-containing TBS washbuffer. As with the MSD immunoassay, the dilution series of recombinant calbindin D28K was used as a standard. TBS containing $0.05 \%$ bovine serum antigen (BSA) was used as dilution buffer. Duplicates of both standard and samples were analyzed and $50 \mu \mathrm{L}$ were pipetted per well. After one hour of incubation, the plate was washed twice with washbuffer and $50 \mu \mathrm{L}$ of anti-calbindin D28K polyclonal rabbit antibody, $2 \mu \mathrm{g} / \mathrm{mL}$, was added per well. After one hour of incubation, the plate was washed twice with wash-buffer and $50 \mu \mathrm{L}$ R-Phycoerythrin- (RPE-) conjugated goat anti-rabbit antibody (Pierce), $1.25 \mu \mathrm{g} / \mathrm{mL}$, was added per well. The plate was then incubated 30 minutes and washed twice with wash-buffer. Finally $150 \mu \mathrm{L}$ of sheath buffer (Bio-Rad, Hercules, Calif, USA) was added to each well and incubated for 10 minutes. The plate was measured on the Luminex ${ }^{100}$ (Luminex Corporation, Austin, Tex, USA) and analyzed using Bio-Plex Manager 3.0 (Bio-Rad). The analysis software was set to acquire data using $100 \mu \mathrm{L}$ of sample per well and to collect a total of 100 beads. The standard curve was calculated using a 5-parameter logistic fit curve generated from 7 standards.

\section{Results and Discussion}

The need for multiple biomarker analysis in limited sample volumes as well as the demand of decreasing costs has led to the development of multiplex immunoassay techniques. Several studies have previously compared the analysis of different biomarkers on different commercially available multiplex platforms [7-11]. Using the same antibody pair, in this study we compared the analysis of a distal tubule-specific protein on the MSD and Luminex platforms.

Recombinant calbindin D28K was used as a standard and was diluted to produce 8- versus 7-standard concentrations for the MSD and Luminex immunoassay, respectively. Figure 1 depicts the standard curves for both MSD and Lumniex measurement of calbindin D28K. The software used in MSD analysis regarded the lowest concentration $(25 \mathrm{pg} / \mathrm{mL})$ on standard curve to be below detection range. The signal coefficient of variation $(\mathrm{cv})$ was less than $10 \%$ for both platforms, while the calculated concentration $\mathrm{cV}$ was high for the lower concentrations on the standard curves. MSD displayed a wider dynamic range than Luminex, detecting concentrations at a maximum of $500 \mathrm{ng} / \mathrm{mL}$ and a minimum of $50 \mathrm{pg} / \mathrm{mL}$. The high calculated concentration $\mathrm{cv}$ at $50 \mathrm{pg} / \mathrm{mL}$, set calbindin D28K lower limit of detection (lld) to $100 \mathrm{pg} / \mathrm{mL}$ on the Luminex platform.

The earliest report on a calbindin D28K immunoassay, made use of anti-calbindin D28K-coupled Sepharose beads and the standard used was native calbindin D28K purified from human kidney tissue [12]. Similar to the Luminex assay, this assay had a lld equivalent to $100 \mathrm{pg} / \mathrm{mL}$. While the earlier-described immunoassay detected calbindin D28K in healthy individuals [5], urinary analysis of both normal rats and human indicated that the calbindin D28K level was below the detection limit in both the MSD and Luminex immunoassay (data not shown). Furthermore, it was not possible to accurately measure urinary spiked recombinant calbindin $\mathrm{D} 28 \mathrm{~K}$, due to the recombinant protein instability in urine. 


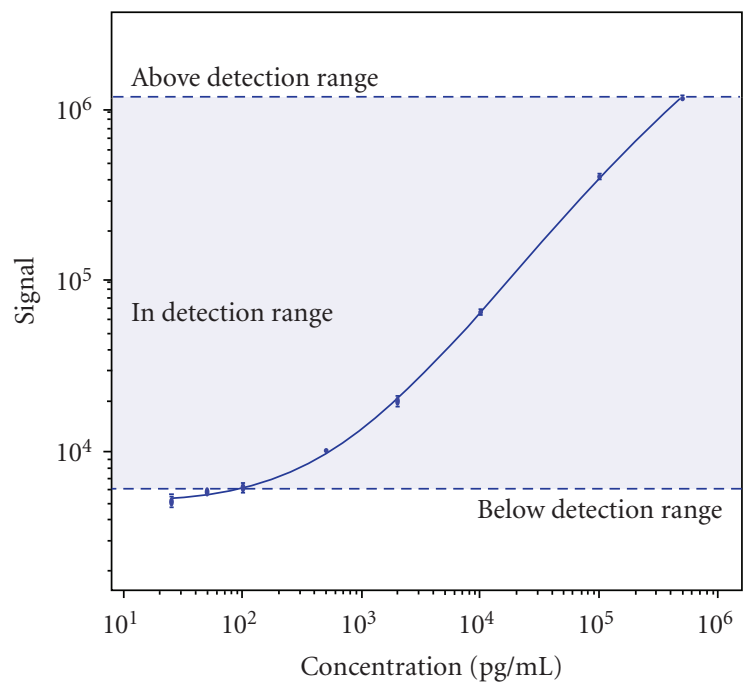

\begin{tabular}{lccccc}
\hline $\begin{array}{l}\text { Concentration } \\
\mathrm{pg} / \mathrm{mL}\end{array}$ & Signal & CV \% & $\begin{array}{c}\text { Cal. } \\
\text { Conc. } \\
\mathrm{pg} / \mathrm{mL}\end{array}$ & $\begin{array}{c}\text { Cal. } \\
\text { Conc. } \\
\text { CV \% }\end{array}$ & \% Recovery \\
\hline 500000 & 1199842 & 3.2 & 495924 & 5.7 & 99 \\
100000 & 417723 & 5.2 & 102364 & 7.0 & 102 \\
10000 & 65565 & 4.2 & 9872 & 5.3 & 99 \\
2000 & 19791 & 6.3 & 1900 & 9.9 & 95 \\
500 & 10478 & 2.2 & 588 & 5.0 & 118 \\
100 & 6162 & 6.0 & 83 & 44 & 83 \\
50 & 5826 & 4.4 & 51 & 47 & 101 \\
\hline
\end{tabular}

(a)

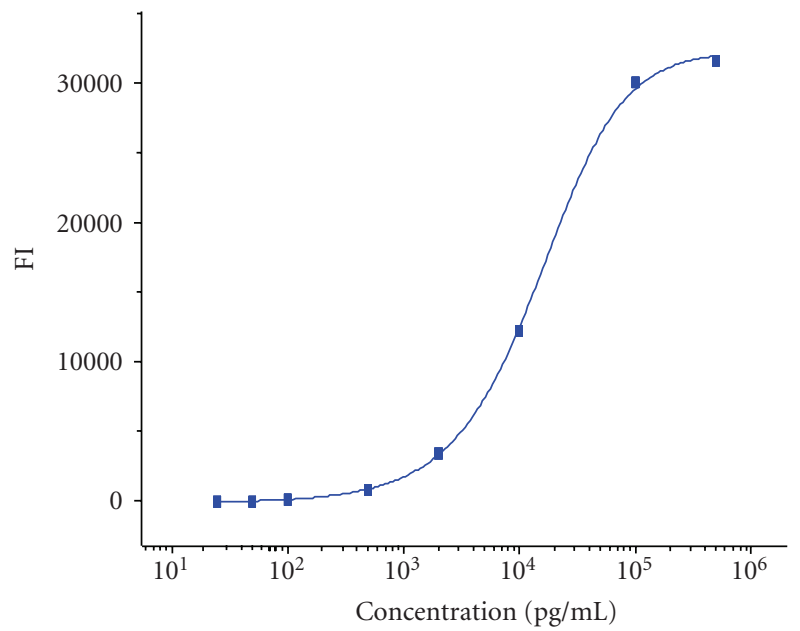

\begin{tabular}{lccccc}
\hline $\begin{array}{l}\text { Concentration } \\
\text { pg/mL }\end{array}$ & Signal & CV \% & $\begin{array}{c}\text { Cal. } \\
\text { Conc. } \\
\text { pg/mL }\end{array}$ & $\begin{array}{c}\text { Cal. } \\
\text { Conc. } \\
\text { CV \% }\end{array}$ & \% Recovery \\
\hline 500000 & 31933 & 1.0 & 294542 & 0.9 & 59 \\
100000 & 30354 & 1.0 & 117304 & 0.7 & 117 \\
10000 & 12562 & 9.0 & 9785 & 9.5 & 98 \\
2000 & 3748 & 2.0 & 2059 & 1.8 & 103 \\
500 & 1215 & 1.0 & 489 & 1.9 & 98 \\
100 & 493 & 2.0 & 106 & 5.8 & 106 \\
50 & 356 & 14 & 42 & 70 & 84 \\
\hline
\end{tabular}

(b)

Figure 1: (a) Calbindin D28K standard curve analysis on MSD. (b) Luminex analysis of calbindin D28K; with standard curve indicating the fluorescence intensity (FI) at the different concentrations.

TABLE 1: Linearity analysis of rat cortical kidney homgenate dilutions.

\begin{tabular}{|c|c|c|c|c|c|c|}
\hline \multirow[b]{2}{*}{ Dilution factor } & \multicolumn{3}{|c|}{ MSD } & \multicolumn{3}{|c|}{ Luminex } \\
\hline & Measured $\mathrm{pg} / \mathrm{mL}$ & $\mathrm{CV}^{+}{ }^{+}$ & \%Mean* & Measured pg/mL & $\mathrm{CV}^{+}{ }^{+}$ & \%Mean* \\
\hline 100 & 5554 & 1.2 & 131 & 49762 & 0.1 & 128 \\
\hline 200 & 2410 & 1.9 & 114 & 22802 & 5.1 & 117 \\
\hline 400 & 1233 & 11 & 116 & 11568 & 1.2 & 119 \\
\hline 800 & 424 & 12 & 80 & 5421 & 8.0 & 112 \\
\hline 1600 & 247 & 6.7 & 93 & 2709 & 5.2 & 111 \\
\hline 3200 & 110 & 16 & 83 & 1120 & 3.7 & 92 \\
\hline 6400 & 55 & 14 & 83 & 422 & 12 & 69 \\
\hline 12800 & - & & & 155 & 6.2 & 51 \\
\hline
\end{tabular}

* Calculated concentration (dilution factor $\times$ measured concentration) as a percentage of the average of the calculated concentration from all dilutions. The average calculated concentration of rat cortical kidney homogenate analyzed by MSD and Luminex was $424 \mathrm{ng} / \mathrm{mL}$ and $3.9 \mu \mathrm{g} / \mathrm{mL}$, respectively.

${ }^{+}$Coefficient of variation. 
TABLE 2: Precision analysis using rat cortical kidney homogenate.

\begin{tabular}{lcccc}
\hline & \multicolumn{2}{c}{ MSD } & \multicolumn{2}{c}{ Luminex } \\
\hline Dilution & $1: 400$ & $1: 4000$ & $1: 400$ & $1: 4000$ \\
Mean & 945 & 87 & 14167 & 1041 \\
$\mathrm{SD}^{*}$ & 97 & 17 & 723 & 103 \\
$\mathrm{CV}^{+}$ & $10 \%$ & $20 \%$ & $5 \%$ & $10 \%$ \\
\hline
\end{tabular}

* Standard of deviation.

${ }^{+}$Coefficient of variation.

TABLE 3: Interassay variation analysis of rat cortical kidney homogenate.

\begin{tabular}{lcc}
\hline Assay & MSD $(\mathrm{ng} / \mathrm{mL})$ & Luminex $(\mu \mathrm{g} / \mathrm{mL})$ \\
\hline 1 & 424 & 3.9 \\
2 & 407 & 3.1 \\
3 & 283 & 3.3 \\
SD $^{*}$ & 76 & 0.4
\end{tabular}

*Standard of deviation.

TABLE 4: MSD specificity analysis of human kidney homogenate.

\begin{tabular}{lc}
\hline Region & Concentration $(\mathrm{ng} / \mathrm{mL})$ \\
\hline Cortex & 64 \\
Medulla & 3 \\
Papilla & - \\
\hline
\end{tabular}

In an attempt to assess the immunoassays ability in measuring native calbindin D28K, kidney homogenates were analyzed. Since calbindin D28K is expressed in distal tubuli, rat cortical kidney homogenate was used in validating the immunoassays sensitivity and linearity. Whereas MSD had a higher sensitivity for recombinant calbindin D28K, Luminex detected calbindin D28K in kidney homogenate at the highest dilution factor tested (Table 1). To assess the immunoassays linearity, the calculated concentration (dilution factor $\times$ measured concentration) was expressed as a percentage of the average of the calculated concentration from all dilutions. There was no clear difference in linearity between Luminex and MSD measurement, except at the high kidney homogenate dilutions where lower level of calbindin D28K was measured by the Luminex assay. Furthermore, Luminex detected almost 10-fold the amount of calbindin D28K as compared to MSD. Previous studies comparing different multiplex platforms have also reported quantitive differences in the analysis of different proteins $[7,8]$. The difference in detected amounts of calbindin D28K could be due to technical differences between the two platforms. Immobilization of capture antibodies on beads in Luminex may provide a larger total surface than immobilization of capture antibodies at the bottom of the wells in MSD plates. This in turn may allow for higher accessibility for the capture antibody to interact with calbindin D28K in solution, and subsequently a higher detection.

To investigate the immunoassays measurement of high versus low concentrations of calbindin D28K, two different dilutions of rat cortical kidney homogenate were analyzed. Precision analysis of the two different platforms indicated that Luminex had lower $\mathrm{cv}$ values as compared to MSD, at both high and low concentrations of calbindin D28K (Table 2). To assess the interassay variation in both platforms, the average of the calculated calbindin D28K concentration (dilution factor $\times$ measured concentration) of all the different kidney homogenate dilutions from three different experiments was compared. Despite the higher standard of deviation (SD) value, Luminex displayed a lower interassay variation as compared to MSD (Table 3).

Whereas the recombinant calbindin $\mathrm{D} 28 \mathrm{~K}$ is cloned from rat and the analyses presented so far was with rat kidney homogenate, the antibodies used in this immunoassay crossreacts with human calbind D28K. To test the specificty of the immunoassay, homogenates from different regions of the human kidney were analyzed with MSD. The result demonstrated the immunoassays specifcity to the predominant expression of the distal tubule calbindin $\mathrm{D} 28 \mathrm{~K}$ in the cortical region (Table 4).

\section{Conclusions}

The methods described herein will enable future multiplex analysis of calbindin D28K in combination with other kidney-damage biomarkers, which may then be used in investigating distal tubule damage in rat and human toxicological studies. Whereas MSD had a wider dynamic range, analysis of calbindin D28K indicated that Luminex has higher sensitivity and precision. The selection of the platform most suitable for kidney biomarker analysis will depend on the other analytes available and other practical factors such as cost and ease of analysis.

\section{Acknowledgments}

This work was supported by the Technology Strategy Board in the United Kingdom.

\section{References}

[1] C. U. Niemann and N. J. Serkova, "Biochemical mechanisms of nephrotoxicity: application for metabolomics," Expert Opinion on Drug Metabolism and Toxicology, vol. 3, no. 4, pp. 527-544, 2007.

[2] K. Sooy, J. Kohut, and S. Christakos, "The role of calbindin and 1,25dihydroxyvitamin $\mathrm{D}_{3}$ in the kidney," Current Opinion in Nephrology and Hypertension, vol. 9, no. 4, pp. 341-347, 2000.

[3] J. Roth, D. Brown, A. W. Norman, and L. Orci, "Localization of the vitamin D-dependent calcium-binding protein in mammalian kidney," American Journal of Physiology, vol. 12, no. 3, pp. F243-F252, 1982.

[4] G. R. Betton, K. Kenne, R. Somers, and A. Marr, "Protein biomarkers of nephrotoxicity; a review amd findings with cyclosporin A, a signal transduction kinase inhibitor and $\mathrm{N}$ phenylanthranilic acid," Cancer Biomarkers, vol. 1, no. 1, pp. 59-67, 2005.

[5] M. Takashi, Y. Zhu, K. Miyake, and K. Kato, "Urinary 28$\mathrm{kD}$ calbindin-D as a new marker for damage to distal renal tubules caused by cisplatin-based chemotherapy," Urologia Internationalis, vol. 56, no. 3, pp. 174-179, 1996. 
[6] S. Hasegawa, K. Kato, M. Takashi, et al., "Increased levels of calbindin-D in serum and urine from patients treated by extracorporeal shock wave lithotripsy," The Journal of Urology, vol. 149, no. 6, pp. 1414-1418, 1993.

[7] S. S. Khan, M. S. Smith, D. Reda, A. F. Suffredini, and J. P. McCoy Jr., "Mulitplex based array assays for detecting of soluble cytokines: comparison of sensitivity and quantative values among kits from multiple manufacturers," Cytometry Part B, vol. 61, no. 1, pp. 35-39, 2004.

[8] F. Chowdhury, A. Williams, and P. Johnson, "Validation and comparison of two multiplex technologies, Luminex ${ }^{\circledR}$ and Mesoscale Discovery, for human cytokine profiling," Journal of Immunological Methods, vol. 340, no. 1, pp. 55-64, 2009.

[9] G. Toedter, K. Hayden, C. Wagner, and C. Brodmerkel, "Simultaneous detection of eight analytes in human serum by two commercially avaliable platforms for multiplex cytokine analysis," Clinical and Vaccine Immunology, vol. 15, no. 1, pp. 42-48, 2008.

[10] M. Y. Liu, A. M. Xydakis, R. C. Hoogeveen, et al., "Mulitplexed analysis of biomarkers related to obesity and the metabolic syndrome in human plasma, using the Luminex-100 system," Clinical Chemistry, vol. 51, no. 7, pp. 1102-1109, 2005.

[11] G. E. Lash, P. J. Scaife, B. A. Innes, et al., "Comparison of three multiplex cytokine analysis systems: Luminex, SearchLight ${ }^{\mathrm{TM}}$ and FAST Quant ${ }^{\circledR}$," Journal of Immunological Methods, vol. 309, no. 1-2, pp. 205-208, 2006.

[12] Y. Zhu, M. Takashi, K. Miyake, and K. Kato, "Sensitive enzyme immunoassay for human $28 \mathrm{kDa}$ calbinin-D," Clinica Chimica Acta, vol. 201, no. 3, pp. 183-192, 1991. 

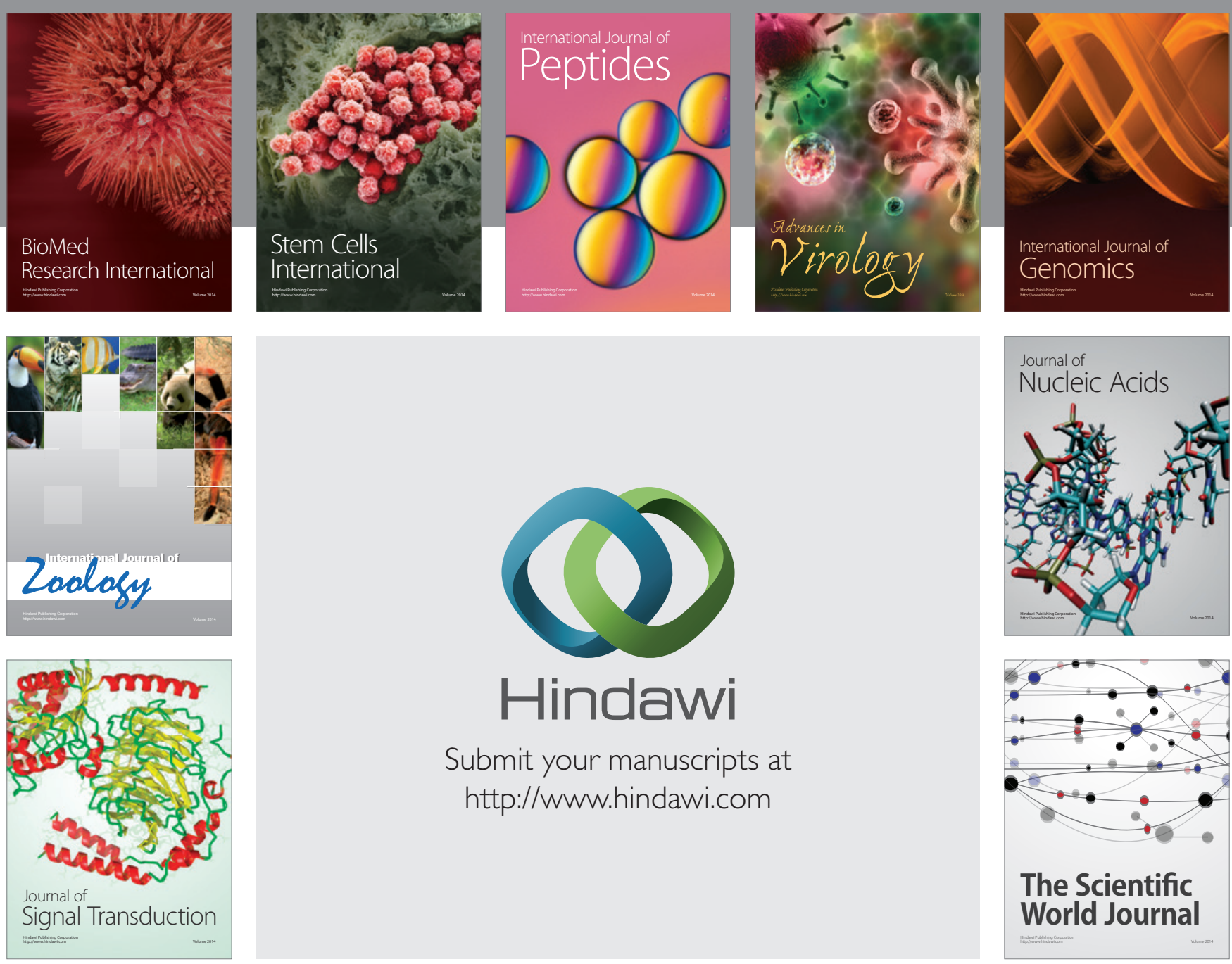

Submit your manuscripts at

http://www.hindawi.com
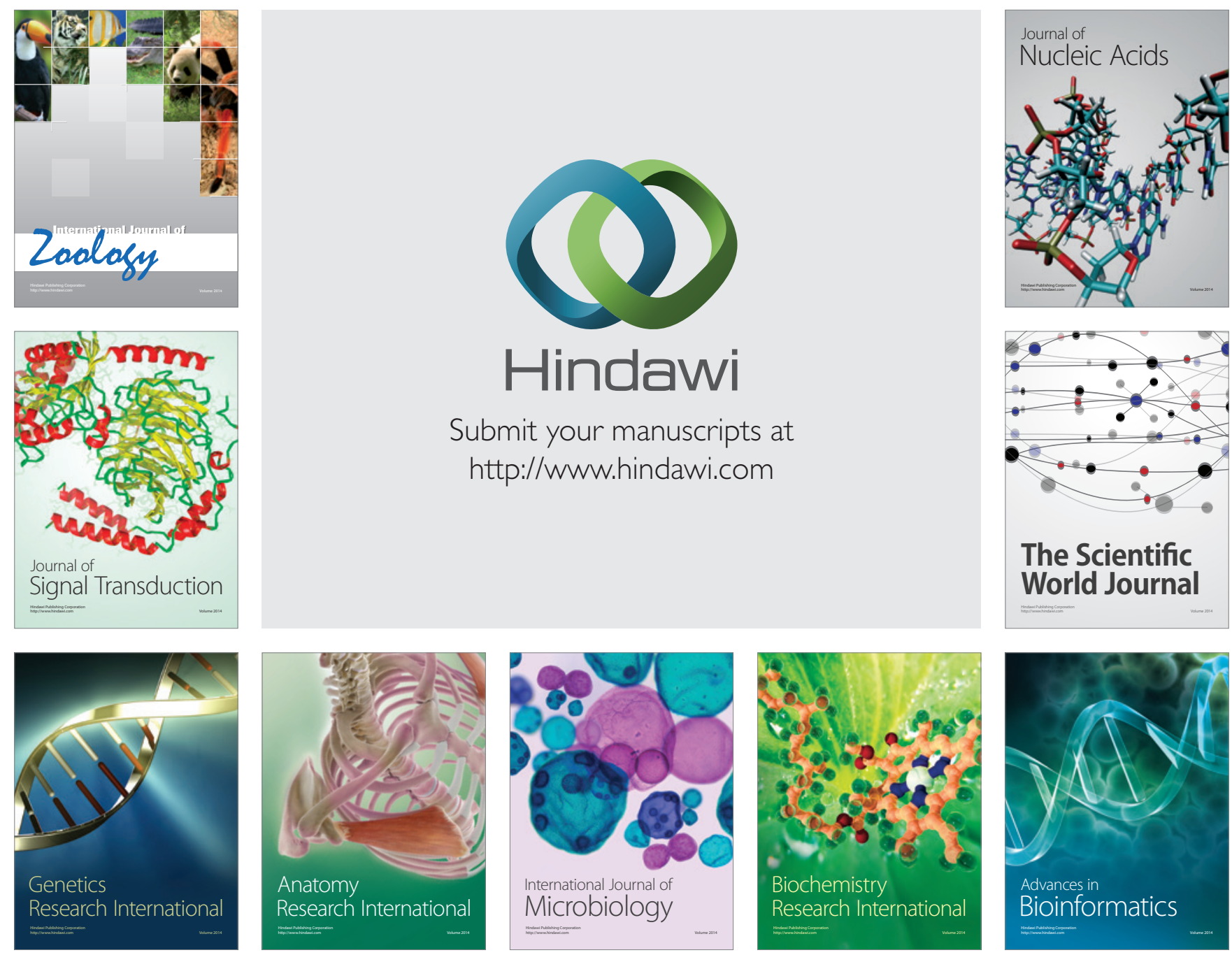

The Scientific World Journal
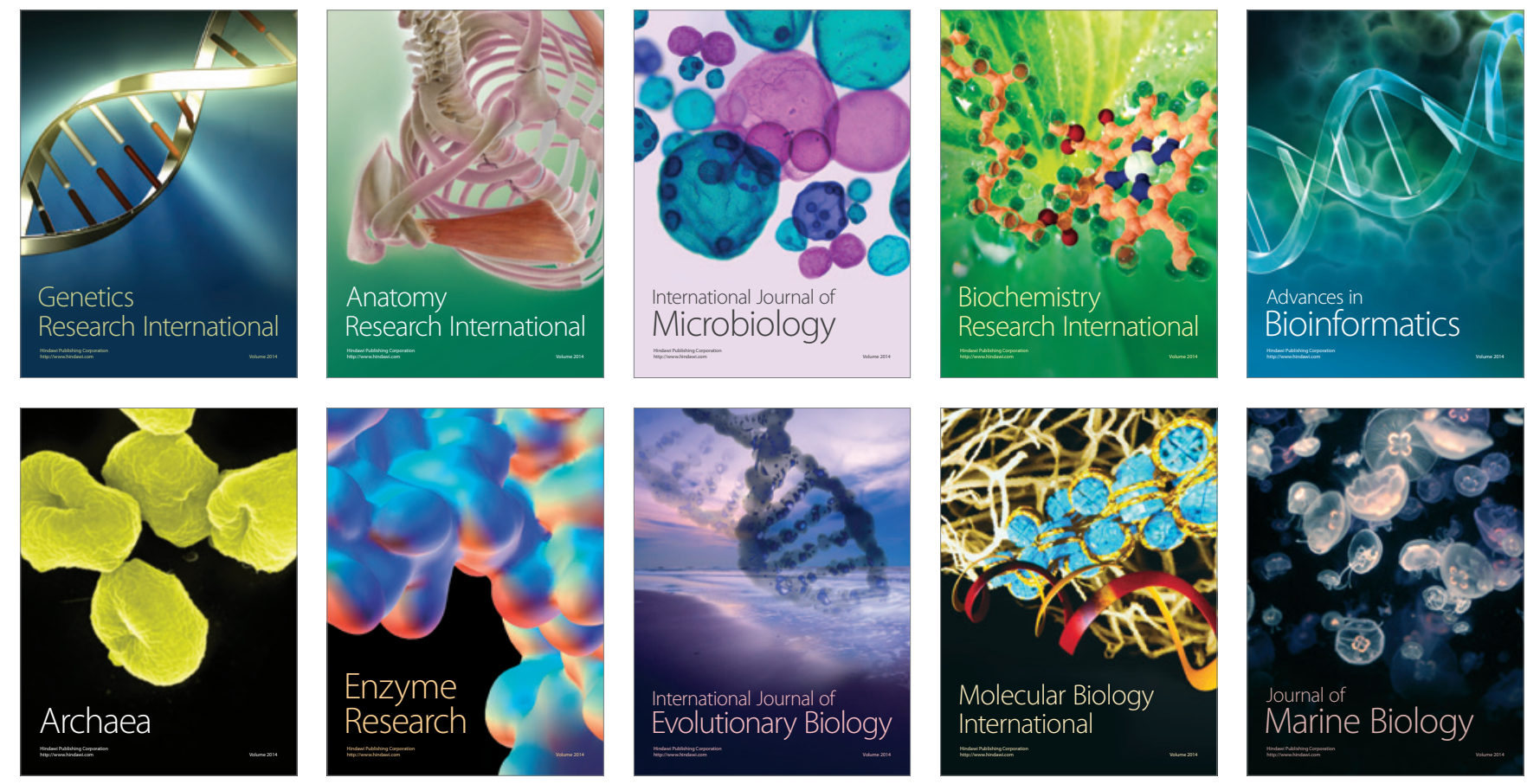